http://dx.doi.org/10.32929/2446-8355.2018v27n4p463-470

\title{
RESPOSTA FISIOLÓGICA DE SEMENTES DE ALCACHOFRA AO ESTRESSE SALINO
}

\author{
Raquel Stefanello $^{1 *}$, Patrícia Carine Hüller Goergen ${ }^{2}$, Luiz Augusto Salles das Neves ${ }^{3}$
}

\author{
${ }^{1}$ Bióloga, Departamento de Biologia, Universidade Federal de Santa Maria, Santa Maria, RS. *E-mail do autor \\ correspondente: raquelstefanello@yahoo.com.br \\ 2 Doutoranda do programa de Pós-graduação em Agronomia, Departamento de Fitotecnia, Universidade Federal \\ de Santa Maria, Santa Maria, RS. \\ ${ }^{3}$ Professor, Departamento de Biologia, Universidade Federal de Santa Maria, Santa Maria, RS
}

Recebido: 05/02/2018; Aceito: 30/11/2018

RESUMO: O excesso de sais na água ou no solo é um dos principais fatores ambientais que afeta a germinação das sementes e diminui a produtividade das culturas. Assim, o objetivo deste estudo foi avaliar a resposta fisiológica das sementes de alcachofra ao estresse salino. $\mathrm{O}$ experimento foi conduzido em câmara B.O.D., na temperatura constante de $20{ }^{\circ} \mathrm{C}$ e em presença de luz. As sementes foram posicionadas sobre papel embebido em solução aquosa de cloreto de cálcio $\left(\mathrm{CaCl}_{2}\right)$ e cloreto de sódio $(\mathrm{NaCl})$ nos potenciais osmóticos correspondentes a zero (água destilada); $-0,10 ;-0,20 ;-0,30 ;-0,40 \mathrm{MPa}$. Os parâmetros avaliados foram: germinação e primeira contagem de germinação (vigor). A redução do potencial osmótico do substrato promoveu decréscimo significativo na germinação e no vigor das sementes de alcachofra. Conclui-se que as sementes desta espécie são sensíveis à salinidade e não toleram potenciais osmóticos iguais ou inferiores a $-0,10 \mathrm{MPa}$, sendo possível o seu cultivo em áreas agrícolas nas concentrações acima destes potenciais.

Palavras-chave: Cynara scolymus L. Salinidade. Germinação. Potencial osmótico.

\section{PHYSIOLOGICAL RESPONSE OF ARTICHOKE SEEDS TO SALT STRESS}

\begin{abstract}
The excess of salts in water or soil is one of the main environmental factors that affect seed germination and decrease the productivity of crops. The objective of this work was to evaluate the physiological response of artichoke seeds to salt stress. The experiment was conducted in a B.O.D. chamber at a constant temperature of $20^{\circ} \mathrm{C}$ and in the presence of light. The seeds were placed on paper soaked with aqueous solutions of calcium chloride $\left(\mathrm{CaCl}_{2}\right)$ and sodium chloride $(\mathrm{NaCl})$ at osmotic potentials equivalent to zero (distilled water), $-0.10,-0.20,-0.30$, and $-0.40 \mathrm{MPa}$. The evaluated parameters were: germination e germination first count (vigor). The reduction of the osmotic potential of the substrate promoted a significant decrease in the germination and vigor of the artichoke seeds. In conclusion, seeds of this species are sensitive to salinity and are not able to tolerate osmotic potentials equal or lower than $-0.10 \mathrm{MPa}$, being possible to cultivate them in agricultural areas with concentrations above these potentials.
\end{abstract}

Key words: Cynara scolymus L. Salinity. Germination. Osmotic potential. 


\section{INTRODUÇÃO}

Os estresses abióticos em espécies vegetais têm despertado grande interesse dos pesquisadores, que buscam cultivares mais produtivas no intuito de auxiliar na adequada recomendação para o plantio ou semeadura sob condições desfavoráveis de cultivo. Os estresses decorrentes do excesso de sais no solo são os mais estudados, em razão de seu impacto sobre o crescimento, desenvolvimento e a produtividade das plantas. A salinidade pode ser responsável por alterações morfológicas, fisiológicas e bioquímicas, com reflexos sobre o transporte de água e nutrientes nas plantas (MUNNS, 2011; FILIPPOU et al., 2014).

Estudos têm sido conduzidos com o uso de soluções salinas em diferentes concentrações, e demonstram que a salinidade apresenta efeito negativo sobre a germinação e emergência de várias culturas, tais como quiabo - Abelmoschus esculentus (DKHIL et al., 2014), rabanete - Raphanus sativus (SARKER et al., 2014), funcho - Foeniculum vulgare (SOLIMAN; EL-SHAIENY, 2014), alcachofra - Cynara scolymus (JORENUSH; RAJABI, 2015), chia - Salvia hispanica (STEFANELLO et al., 2015), aveia - Hordeum vulgare (ASKARI et al., 2016), feijão caupi - Vigna sinensis (KANDIL et al., 2017), entre outros. De maneira geral, estes autores sugerem que a redução do potencial osmótico do substrato afeta negativamente a germinação das sementes de acordo com a espécie e a concentração da solução utilizada.

Neste contexto, a semente de alcachofra (Cynara scolymus L.) foi escolhida para este estudo por apresentar poucos trabalhos científicos disponíveis relacionados ao desenvolvimento em condições de estresse salino, além de grande importância nutricional e medicinal. Esta espécie, cultivada via sementes, pertence à família Asteraceae sendo originária da região mediterrânica da Europa e produzida em todo o mundo. A demanda como planta medicinal tem aumentado durante as últimas décadas devido à grande aceitação de suas folhas e inflorescências com propósitos nutritivos e para o tratamento de várias doenças, em preparações fitoterápicas com indicação especial para afecções hepáticas e urinárias (EICH et al., 2005; ALONSO et al. 2006; KÜSKÜ-KIRAZ et al., 2010).

Considerando a importância econômica e medicinal desta espécie e que as informações sobre os efeitos da salinidade na germinação das sementes podem contribuir para novos conhecimentos sobre a fisiologia da cultura, objetivou-se avaliar a resposta fisiológica das sementes de alcachofra ao estresse salino.

\section{MATERIAL E MÉTODOS}

O trabalho experimental foi desenvolvido no Laboratório de Genética Vegetal, do Departamento de Biologia, Centro de Ciências Naturais e Exatas da Universidade Federal de Santa Maria (RS). As sementes de alcachofra roxa (Cynara scolymus L.) foram adquiridas de uma empresa tradicional em produção e comercialização de sementes (Feltrin Sementes Farroupilha, RS).

Para as avaliações do efeito do estresse salino, utilizou-se solução aquosa de cloreto de cálcio $\left(\mathrm{CaCl}_{2}\right)$ e cloreto de sódio $(\mathrm{NaCl})$ nas concentrações correspondentes a zero (controle); 
- 0,10;-0,20; -0,30; -0,40 MPa. O nível zero correspondeu à testemunha, onde se utilizou apenas água destilada. A quantidade da solução necessária para obtenção dos potenciais osmóticos foi obtida pela equação de Van’t Hoff citada por Taiz e Zeiger (2013) e por Braccini et al. (1996).

O potencial fisiológico das sementes foi avaliado em câmara de germinação do tipo Biochemical Oxigen Demand (B.O.D.), com regime de $8 \mathrm{~h}$ de luz e $16 \mathrm{~h}$ sem luz, por meio do teste de germinação realizado em caixas plásticas transparentes (gerbox). As sementes foram acondicionadas sobre três folhas de papel germitest ${ }^{\circledR}$ previamente umedecidas com água destilada ou com as respectivas soluções salinas na proporção de 2,5 vezes o peso seco do papel. Após a semeadura, as caixas plásticas foram mantidas na temperatura de $20{ }^{\circ} \mathrm{C}$, sendo a primeira contagem de germinação realizada aos sete dias e a última contagem aos 21 dias. Foram avaliadas as plântulas normais, sendo os resultados expressos em percentagem (BRASIL, 2009).

O delineamento experimental utilizado foi o inteiramente casualizado, onde os tratamentos foram constituídos pelos diferentes sais e diferentes concentrações das soluções, em esquema bifatorial ( $2 \times 5)$, com quatro repetições de 50 sementes. As variáveis percentagem de germinação e primeira contagem foram transformadas em arc sen $\sqrt{x} / 100$. Os dados foram submetidos à análise de variância pelo teste $\mathrm{F}$ e, quando constatado efeito significativo, foi efetuada a análise de regressão pelo programa Sisvar (FERREIRA, 2011).

\section{RESULTADOS E DISCUSSÃO}

A análise de variância indicou que, na germinação e na primeira contagem, houve efeito significativo dos sais e das concentrações utilizadas. Além disso, para a variável germinação houve interação entre os tipos de sais e as diferentes concentrações.

$\mathrm{Na}$ ausência de sais, as sementes apresentaram, em média, germinação de 77\%, verificando-se redução acentuada e diretamente proporcional com incremento das concentrações salinas, obtendo-se baixa germinação (21\%) a partir do potencial -0,20 MPa na solução de $\mathrm{CaCl}_{2}$. Por outro lado, as sementes submetidas ao $\mathrm{NaCl}$ apresentaram $55 \%$ de germinação no menor potencial osmótico utilizado (-0,40 MPa). O efeito do cloreto de cálcio sobre a germinação das sementes foi mais acentuado que o cloreto de sódio, embora fosse verificado que independente do sal utilizado ocorreu a redução da percentagem de germinação, conforme aumentou o estresse salino (Figura 1).

De acordo com Taiz e Zeiger (2013), o aumento da salinidade acarreta mudanças na capacidade da planta em absorver, transportar e utilizar os íons necessários ao seu crescimento e reduz a atividade de enzimas responsáveis pela respiração e fotossíntese. Desta forma, a obtenção de energia para o crescimento e diferenciação das células em tecidos é restringida, limitando o alongamento do eixo embrionário. Além disso, o efeito prejudicial ocorre devido ao estresse osmótico e à toxicidade, consequentemente, a interação de efeitos iônicos e osmóticos específicos conduzem a uma queda na taxa de germinação das sementes (KAZEMI; ESKANDARI, 2011). 


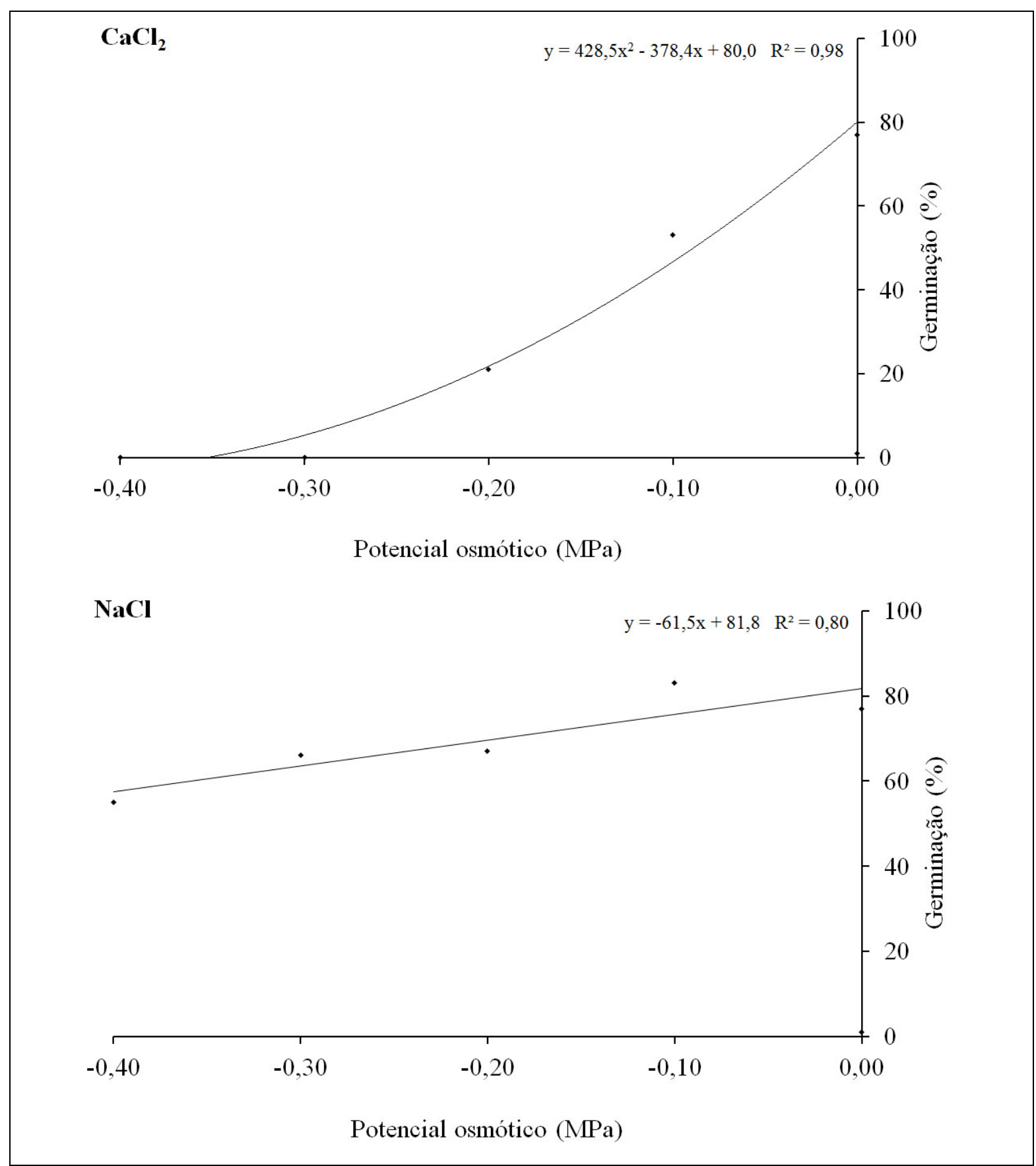

Figura 1. Percentagem de germinação de sementes de alcachofra submetidas a diferentes concentrações de sais $\left(\mathrm{CaCl}_{2} \mathrm{e} \mathrm{NaCl}\right)$. Percentage of germination of artichoke seeds exposed to different concentrations of salts $\left(\mathrm{CaCl}_{2}\right.$ e $\left.\mathrm{NaCl}\right)$.

Os resultados deste estudo corroboram com os realizados em beterraba - Beta vulgaris (MACIEL et al., 2015), verificando-se redução na porcentagem de germinação, com o aumento da concentração salina no meio de cultivo. Adicionalmente, o potencial hídrico a partir de -0,8 MPa torna-se uma condição limitante na germinação e formação de plântulas normais de catingueira (Poincianela pyramidalis) e angico (Anadenanthera colubrina), sendo este efeito mais evidente quando utilizado o $\mathrm{CaCl}_{2}$ (SANTOS et al., 2016). Da mesma forma, Jorenush e Rajabi (2015) observaram que, em sementes de alcachofra, o decréscimo do potencial osmótico do substrato reduziu consideravelmente a taxa de germinação, o comprimento e o peso seco da parte aérea e da raiz. 


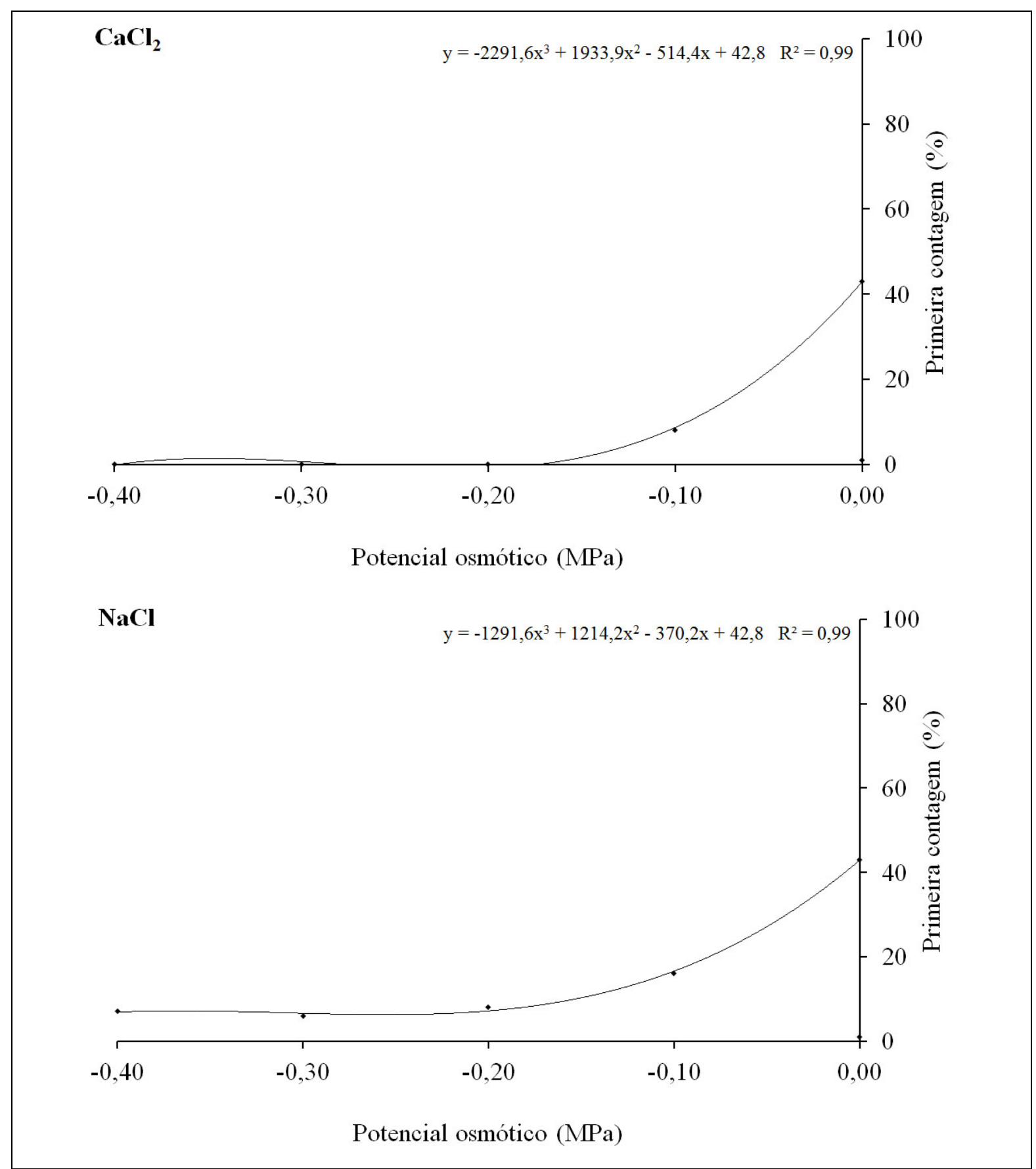

Figura 2. Primeira contagem de germinação de sementes de alcachofra submetidas a diferentes concentrações de sais. First germination count of artichoke seeds exposed to different concentrations of salts $\left(\mathrm{CaCl}_{2}\right.$ e $\left.\mathrm{NaCl}\right)$.

O vigor das sementes, avaliado pelo teste de primeira contagem de germinação, foi reduzido com o aumento da concentração das soluções salinas (Figura 2). Observou-se redução acentuada da percentagem de plântulas normais a partir do potencial de $-0,10 \mathrm{MPa}$ nos dois agentes indutores de déficit hídrico $\left(\mathrm{CaCl}_{2}\right.$ e $\left.\mathrm{NaCl}\right)$. Além disso, não foram observadas plântulas normais a partir do potencial -0,20 MPa quando utilizadas as soluções de $\mathrm{CaCl}_{2}$.

De acordo com Pallardy (2008), entre os agentes osmóticos, o sal $\mathrm{CaCl}_{2}$ provoca maior efeito negativo no desenvolvimento das plantas devido ao $\mathrm{Ca}^{2+}$ ser um mensageiro secundário 
da transdução de sinais, sendo que pequenas variações na sua concentração acabam alterando as atividades das enzimas ligadas a este nutriente.

Além disso, o excesso de sal causa diminuição da captação de água devido ao decréscimo do potencial osmótico do substrato, prolongando a embebição das sementes ou o alongamento da raiz, além do efeito tóxico nos tecidos devido à grande quantidade de íons $\mathrm{Na}^{+}$e $\mathrm{Cl}^{-}$(ESTEVES; SUZUKI, 2008; CHAVES et al., 2009).

Utilizando outras soluções que também simulam o estresse salino em laboratório, Jamil et al. (2006) observaram que o aumento da concentração de cloreto de sódio $(\mathrm{NaCl})$ causou redução na porcentagem e velocidade de germinação, no comprimento de raiz e de parte aérea em sementes de repolho (Brassica oleracea capitata), amaranto (Amaranthus paniculatus), beterraba (Beta vulgaris) e mostarda (Brassica campestris). Também, Kandil et al. (2017) utilizando altas concentrações de soluções salinas $(\mathrm{NaCl})$ constataram decréscimo da germinação, do vigor e do comprimento da raiz e da parte aérea das sementes de feijão caupi (Vigna sinensis).

Vale destacar que os efeitos do estresse diferem durante o ciclo de vida da planta de modo que aqueles verificados em sementes nem sempre são os mesmos observados em plantas em desenvolvimento. Desta forma, sob qualquer estresse ambiental exercido, o efeito vai depender de cada planta, da sua adaptação, concentração e duração da exposição do indivíduo frente a este fator (KRANNER et al., 2010). Por fim, os resultados deste estudo demonstraram que as sementes de alcachofra são sensíveis à exposição a diferentes sais nos potenciais osmóticos compreendidos entre - 0,10 e - - , 40 $\mathrm{MPa}$, sendo que o efeito do sal $\mathrm{CaCl}_{2}$ sobre a germinação das sementes foi mais acentuado que o $\mathrm{NaCl}$. Possivelmente, a disponibilidade de água seja um fator limitante para a germinação de sementes de alcachofra. Estas informações podem auxiliar no entendimento da distribuição geográfica desta espécie e no papel que a escassez de água desempenha na germinação de suas sementes, contribuindo com novos conhecimentos sobre a fisiologia dessa cultura.

\section{CONCLUSÃO}

As sementes de alcachofra são sensíveis à salinidade. Potenciais osmóticos iguais ou inferiores a -0,10 MPa são prejudiciais para a germinação e o vigor de sementes de alcachofra.

\section{REFERÊNCIAS BIBLIOGRÁFICAS}

ALONSO, M.R.; GARCIA, M.C.; BONELLI, C.G. Validated HPLC method for cynarin determination in biological sample. Acta Farmacéutica Bonaerense, La Plata, v. 25, n. 2, p.267-70, 2006.

ASKARI, H.; KAZEMITABAR, S.K.; ZARRINI, H.N.; SABERI, M.H. Salt tolerance assessment of barley (Hordeum vulgare L.) genotypes at germination stage by tolerance indices. Open Agriculture, Warsaw, v. 1, n. 1, p.37-44, 2016. 
BAIER, C.; EICH, J.; GRUN, M.; WAGENBRETH, D.; ZIMMERMANN, R. Artichoke leaves used for herbal drug production: Influence of nitrogen fertilization on yield and on pharmaceutical quality. Acta Horticulturae, The Hague, v. 681, n. 1, p.545-551, 2005.

BRACCINI, A.L.; BRACCINI, A.L.; RUIZ, H.A.; BRACCINI, M.C.L.; REIS, M.S. Germinação e vigor de sementes de soja sob estresse hídrico induzido por soluções de cloreto de sódio, manitol e polietilenoglicol. Revista Brasileira de Sementes, Londrina, v. 18, n. 1, p.10-16, 1996.

BRASIL. Ministério da Agricultura, Pecuária e Abastecimento. Secretaria de Defesa Agropecuária. Regras para análise de sementes. Brasília: Mapa/ACS, 2009. 399 p.

CHAVES, M.M.; FLEXAS, J.; PINHEIRO, C. Photosynthesis under drought and salt stress: regulation mechanisms from whole plant to cell. Annals of Botany, Oxford, v. 103, n. 4, p.551-560, 2009.

DKHIL, B.B., ISSA, A., DENDEN, M. Germination and seedling emergence of primed okra (Abelmoschus esculentus L.) seeds under salt stress and low temperature. American Journal of Plant Physiology, Vails Gate, v. 9, n. 2, p.38-45, 2014.

ESTEVES, B.S.; SUZUKI, M.S. Efeito da salinidade sobre as plantas. Oecologia Brasiliensis, Rio de Janeiro, v. 12, n. 4, p.662-679, 2008.

FERREIRA, D.F. Sisvar: a computer statistical analysis system. Ciência e Agrotecnologia, Lavras, v. 35, n. 6, p.1039-1042, 2011.

FILIPPOU, P.; BOUCHAGIER, P.; SKOTTI, E.; FOTOPOULOS, V. Proline and reactive oxygen/nitrogen species metabolism is involved in the tolerant response of the invasive plant species Ailanthus altissima to drought and salinity. Environmental and Experimental Botany, Oxford, v. 97, p.1-10, 2014.

KANDIL, A.A.; SHAREIF, A.E.; GAD, M.A. Effect of salinity on germination and seedling parameters of forage cowpea seed. Research Journal of Seed Science, USA, v. 10, n. 1, p.17-26, 2017.

KAZEMI, K., ESKANDARI, H. Effects of salt stress on germination and early seedling growth of rice (Oryza sativa) cultivars in Iran. African Journal of Biotechnology, Nairobi, v. 10, n. 77, p.17789-17792, 2011.

KRANNER, I.; MINIBAYEVA, F.V.; BECKETT, R.P.; SEAL, C.E. What is stress? Concepts, definitions and applications in seed science. The New Phytologist, Oxford, v. 188, n. 3, p.655-673, 2010.

KÜSKÜ-KIRAZ, Z.; MEHMETC, I.K.G.; DOGRU-ABBASOGLU, S.; UYSAL, M. Artichoke leaf extract reduces oxidative stress and lipoprotein dyshomeostasis in rats fed on high cholesterol diet. Phytotherapy Research, Chichester, v. 24, n. 4, p.565-570, 2010.

JAMIL, M.; DEOG BAE, L.; KWANG YONG, J.; ASHRAF, M.; HEONG CHUN, L.EUI SHIK, R. Effect of salt $(\mathrm{NaCl})$ stress on germination and early seedling growth of four vegetables species. Journal of Central European Agriculture, Zagreb, v. 7, n. 2, p.273-282, 2006. 
JORENUSH, M.H.; RAJABI, M. Effect of drought and salinity tensions on germination and seedling growth of artichoke (Cynara scolymus L.). International Journal of Advanced Biological and Biomedical Research, Iran, v. 3, n. 3, p.297-302, 2015.

MACIEL, K.S.; MORAES, J.C.; FARIAS, C.E.; MAGALHÃES, C.C.M.; LIMA, P.A.M. Germinação de sementes de beterraba em condições de estresse hídrico e salino. Nucleus, Ituverava, v. 12, n. 2, p.189-200, 2015.

MUNNS, R. Plant adaptations to salt and water stress: differences and commonalities. In: TURKAN, I. (Ed.). Plant responses to drought and salinity stress: developments in a postgenomic Era. London: Elsevier, 2011.p. 132. (Advances in Botanical Research, 57).

PALLARDY, D.S.G. Molecules physiology of woody plants (Third Edition). San Diego: Academic Press, 2008. 377 p.

SANTOS, C.A.; SILVA, N.V.; WALTER, L.S.; SILVA, E.C.A.; NOGUEIRA, R.J.M.C. Germinação de sementes de duas espécies da caatinga sob déficit hídrico e salinidade. Pesquisa Florestal Brasileira, Colombo, v. 36, n. 87, p.219-224, 2016.

SARKER, A.; HOSSAIN, M.D.I.; ABUL KASHEM, M.D. Salinity ( $\mathrm{NaCl})$ tolerance of four vegetable crops during germination and early seedling growth. International Journal of Latest Research in Science and Technology, Yamuna Nagar, v. 3, n. 1, p.91-95, 2014.

SOLIMAN, W.S.; EL-SHAIENY, A.A.H. Effect of saline water on germination and early growth stage of five Apiaceae species. African Journal of Agricultural Research, v. 9, n. 7, p.713-719, 2014.

STEFANELLO, R., ABBAD, M.A.B., NEVES, L.A.S., VIANA, B.B. Resposta fisiológica de sementes de chia (Salvia hispanica - Lamiales: Lamiaceae) ao estresse salino. Biotemas, Florianópolis, v. 28, n. 4, p.35-39, 2015.

TAIZ, L.; ZEIGER, E. Fisiologia vegetal. 5 ed. Porto Alegre: Artimed, 2013. 954 p. 